\title{
Regularity of spherical means
}

J. Peyrière and P. Sjölin

\section{Introduction}

Let $\mathbf{R}^{n}$ denote $n$-dimensional Euclidean space and let $|x|$ denote the norm of an element $x \in \mathbf{R}^{n}$. For $\beta \in \mathbf{R}$ and $f \in L_{\text {loc }}^{\mathbf{1}}\left(\mathbf{R}^{n}\right)$ we set

$$
F_{\beta, x}(t)=|t|^{\beta} \int_{S^{n-1}} f(x-t y) d \sigma(y), \quad x \in \mathbf{R}^{n}, \quad t \in \mathbf{R},
$$

where $\sigma$ denotes the surface measure on $S^{n-1}=\left\{x \in \mathbf{R}^{n} ;|x|=1\right\}$. It follows from Fubini's theorem that for every $x \in \mathbf{R}^{n}, F_{\beta, x}(t)$ is well-defined for almost all $t \in \mathbf{R}$. We also set $F_{x}(t)=F_{0, x}(t), t \geqq 0$, and $F_{x}(t)=0$ for $t<0$.

E. M: Stein [2] has studied the maximal operator $M$ defined by

$$
M f(x)=\sup _{t \geq 0}\left|F_{x}(t)\right|, \quad x \in \mathbf{R}^{n}, \quad f \in \mathscr{P}\left(\mathbf{R}^{n}\right),
$$

where $\mathscr{S}\left(\mathbf{R}^{n}\right)$ denotes the Schwartz class, and has proved that $\|M f\|_{L^{p}\left(\mathbf{R}^{n}\right)} \leqq$ $C_{p}\|f\|_{L^{p}\left(\mathrm{R}^{n}\right)}$ if $n \geqq 3$ and $p>n /(n-1)$.

The purpose of this paper is to study the regularity of the function $F_{\beta, x}$ in terms of Besov (=Lipschitz) spaces. We let the Besov spaces $B_{p}^{\alpha, q}=B_{p}^{\alpha, q}(\mathbf{R})$ be defined as in P. Brenner, V. Thomée and L. B. Wahlbin [1]. These spaces are known to coincide with the Lipschitz spaces $\Lambda_{a}^{p, q}$ studied by M. H. Taibleson [3].

If $f$ is a complex-valued function on $\mathbf{R}^{n}$ we write $\breve{f(x)}=\overline{f(-x)}$. In Sections $2-4$ we obtain the following results.

Theorem 1. Assume $n \geqq 2, \alpha>0$ and $-1<\beta<(n-2) / 2$. Then there exists a constant $C$ such that

$$
\int_{\mathbf{R}^{n}}\left\|F_{\beta, x}\right\|_{B_{2}^{\alpha}, 2(\mathbf{R})}^{2} d x \leqq C \int_{\mathbf{R}^{n}}|f * \breve{f}(x)||x|^{2(\beta-\alpha)-n+1}\left(1+|x|^{2 \alpha}\right) d x
$$

for every continuous function $f$ with compact support in $\mathbf{R}^{n}$.

Corollary 1. Assume $n \geqq 2$ and $1 \leqq p<q \leqq 2$. If $-1<\beta<\inf ((n-2) / 2$, $n(1 / p-1 / 2)-1 / 2), 0<\alpha<\beta+1 / 2-n(1 / q-1 / 2)$ and $f \in L^{p}\left(\mathbf{R}^{n}\right) \cap L^{q}\left(\mathbf{R}^{n}\right)$, then $F_{\beta, x} \in$ $B_{2}^{\alpha, 2}(\mathbf{R})$ for almost every $x \in \mathbf{R}^{n}$. 
Corollary 2. Assume $n \geqq 3, n /(n-1)<q \leqq 2$ and $f \in L_{\text {loc }}^{q}\left(\mathbf{R}^{n}\right)$. If $\alpha<n(1-1 / q)-1$, then for almost every $x \in \mathbf{R}^{n}$ the function $F_{x}$ coincides almost everywhere on ] $0, \infty$ [ with a function which belongs locally to $\Lambda_{\alpha}(] 0, \infty[)$.

In Section 5 we use a different method to prove the following theorem.

Theorem 2. If $n \geqq 3, n /(n-1)<p<\infty, \alpha<1 / p$ and $f \in L^{p}\left(\mathbf{R}^{n}\right)$, then $F_{x} \in B_{p}^{\alpha, 1}(\mathbf{R})$ for almost every $x \in \mathbf{R}^{n}$.

We remark that it is easy to see that Theorem 2 holds also if the function $F_{x}$ is replaced by $F_{0, x}$.

\section{Some lemmas}

To prove Theorem 1 we use the following characterization of the Besov space $B_{2}^{\alpha, 2}(\mathbf{R})$. If $g$ is a function in $L^{2}(\mathbf{R})$ let $u$ denote its Poisson integral. Then $g$ lies in $B_{2}^{\alpha, 2}(\mathbf{R})$ if and only if for some (or every) integer $k>\alpha$ the quantity

$$
\|g\|_{L^{2}(\mathbf{R})}+\left[\int_{0}^{+\infty} y^{2(k-\alpha)-1}\left(\int_{\mathbf{R}}\left|(\partial / \partial y)^{k} u(x, y)\right|^{2} d x\right) d y\right]^{1 / 2}
$$

is finite. This defines equivalent norms on $B_{2}^{\alpha, 2}(\mathbf{R})$.

Let us consider $u_{0}(x, y)=\pi^{-1} y /\left(x^{2}+y^{2}\right)$ the Poisson kernel of the upper half plane. We set $u_{k}=(\partial / \partial y)^{k} u_{0}$.

Since $u_{2 k}$ is homogeneous of degree $-(2 k+1)$ with respect to $(x, y)$ and is an odd function of $y$, there exists a constant $C_{k}$ such that

$$
\left|u_{2 k}(x, y)\right| \leqq C_{k} y /\left(x^{2}+y^{2}\right)^{k+1} \quad\left(x \in \mathbf{R}, y \in \mathbf{R}^{+}\right) .
$$

Lemma 1. We have $u_{k}(., y) * u_{k}(., y)=u_{2 k}(., 2 y)$.

Proof. This lemma is an easy consequence of the formula

$$
\mathscr{F}(u(., y))(\xi)=e^{-|\xi| y} \text {. }
$$

Let us denote by $\sigma_{n}$ the rotation invariant probability measure on $S^{n}$, by $s_{n}$ the area of $S^{n}$ and by $\chi$ the characteristic function of the set $\left\{(x, y, z) \in \mathbf{R}^{3}\right.$; ||$x|-| y||<|z|<|x|+|y|\}$. When $\chi(x, y, z)$ equals 1 we set

$$
\Delta(x, y, z)=\frac{1}{4}\left(\left[(x+y)^{2}-z^{2}\right]\left[z^{2}-(x-y)^{2}\right]\right)^{1 / 2}
$$

(it is the area of the triangle with sides of length $|x|,|y|,|z|$ ).

Lemma 2. Let $n$ be a positive integer, $r$ and $s$ two non-zero real numbers. Let $u s$ denote by $\mu_{n}$ the image measure of $\sigma_{n} \times \sigma_{n}$ by the mapping $(y, z) \mapsto r y+s z$ from $S^{n} \times S^{n}$ to $\mathbf{R}^{n+1}$. We have

$$
d \mu_{n}(x)=\frac{2^{n-2} s_{n-1}}{s_{n}^{2}} \frac{[\Delta(|x|, r, s)]^{n-2}}{(|r s| \cdot|x|)^{n-1}} \chi(|x|, r, s) d x .
$$


Proof. We may suppose $0<r \leqq s$. Let $t$ be a non-negative number. Let us compute $\omega(t)=\mu_{n}(\{x ;|x| \leqq t\})$. We have

$$
\omega(t)=\int_{S^{n}}\left(\int_{S^{n} \cap\{y ;|r y+s z| \leqq t\}} d \sigma_{n}(y)\right) d \sigma_{n}(z) .
$$

The inner integral does not depend on $z$ so we get

$$
\omega(t)=\int_{S^{n} \cap\{y ;|r y+s z| \leqq t\}} d \sigma_{n}(y)
$$

where $z$ is any point in $S^{n}$. Clearly $\omega(t)$ is zero if $t$ is less than $s-r$ and is 1 if $t$ is greater than $s+r$. If $t$ is between $s-r$ and $s+r$ we denote by $\varphi_{0}$ the number such that $0<\varphi_{0}<\pi$ and $t^{2}=r^{2}+s^{2}-2 r s \cos \varphi_{0}$. We then have

so

$$
\omega(t)=\frac{s_{n-1}}{s_{n}} \int_{0}^{\varphi_{0}}(\sin \varphi)^{n-1} d \varphi \quad \text { and } \quad r s \sin \varphi_{0}=2 \Delta(r, s, t)
$$

$$
\omega^{\prime}(t)=2^{n-2} \frac{s_{n-1}}{s_{n}} \frac{t[\Delta(r, s, t)]^{n-2}}{(r s)^{n-1}} \chi(r, s, t) .
$$

The result follows because $\mu_{n}$ is rotation invariant.

Lemma 3. Let $v$ and $w$ be two real numbers such that $v<0, w>-1,2(v+w)<$ -1 . Let us set

$$
\lambda(s)=\left\{\begin{array}{lll}
\int_{1}^{+\infty}\left(t^{2}-s^{2}\right)^{v}\left(t^{2}-1\right)^{w} d t & \text { when } & |s|<1 \\
\int_{0}^{1}\left(s^{2}-t^{2}\right)^{v}\left(1-t^{2}\right)^{w} d t & \text { when } & |s|>1
\end{array}\right.
$$

Then we have, when s tends to 1 ,

$$
\lambda(s)=\left\{\begin{array}{lll}
O(1) & \text { if } & v+w>-1, \\
O(\log (1 /|1-s|)) & \text { if } & v+w=-1, \\
O\left(|1-s|^{v+w+1}\right) & \text { if } & v+w<-1 .
\end{array}\right.
$$

Proof. First let us study the case when $s$ tends to $1^{+}$. If $s$ is less than 2 we have

$$
\begin{aligned}
\lambda(s) & \leqq C \int_{0}^{1}(s-t)^{v}(1-t)^{w} d t=C \int_{0}^{1}(s-1+t)^{v} t^{w} d t \\
& =C(s-1)^{v+w+1} \int_{0}^{1 /(s-1)}(1+t)^{v} t^{w} d t
\end{aligned}
$$

and we conclude easily.

Let us now study the case when $s$ tends to $1^{-}$. We have

$$
\begin{aligned}
\lambda(s) & \leqq \int_{1}^{2}\left(t^{2}-s^{2}\right)^{v}\left(t^{2}-1\right)^{w} d t+\int_{2}^{+\infty}\left(t^{2}-1\right)^{v+w} d t \\
& \leqq C\left(1+\int_{1}^{2}(t-s)^{v}(t-1)^{w} d t\right) \leqq C\left(1+\int_{0}^{1}(1-s+t)^{v} t^{w} d t\right)
\end{aligned}
$$

and we conclude as above. 


\section{Proof of Theorem 1}

Let us compute first $\int_{\mathbf{R}^{n}}\left\|F_{\beta, x}\right\|_{L^{2}\left(\mathbf{R}^{n}\right)}^{2} d x$.

We have

thus

$$
\left|F_{\beta, x}(t)\right|^{2}=|t|^{2 \beta} \iint_{S^{n-1} \times S^{n-1}} f\left(x-t y_{1}\right) \overline{f\left(x-t y_{2}\right)} d \sigma\left(y_{1}\right) d \sigma\left(y_{2}\right),
$$

$$
\int_{\mathbf{R}^{n}}\left|F_{\beta, x}(t)\right|^{2} d x=|t|^{2 \beta} \iint_{S^{n-1} \times S^{n-1}} f * \check{f}\left(-t y_{1}+t y_{2}\right) d \sigma\left(y_{1}\right) d \sigma\left(y_{2}\right) .
$$

Using Lemma 2 we get

therefore

$$
\left.\int_{\mathbf{R}^{n}}\left|F_{\beta, x}(t)^{\mid 2} d x=C\right| t\right|^{2 \beta} \int_{\mathbf{R}^{n}} f * \check{f(x)} \frac{[\Delta(|x|, t, t)]^{n-3}}{\left(t^{2}|x|\right)^{n-2}} \chi(|x|, t, t) d x,
$$

$$
\begin{gathered}
\int_{\mathbf{R}^{n}}\left\|F_{\beta, x}\right\|_{L^{2}(\mathrm{R})}^{2} d x=C \int_{\mathbf{R}^{n}} f * \check{f}(x)\left[\int_{|x| / 2}^{+\infty} \frac{t^{2(\beta-n+2)}}{|x|}\left(4 t^{2}-|x|^{2}\right)^{(n-3) / 2} d t\right] d x \\
=C\left[\int_{1 / 2}^{+\infty} t^{2(\beta-n+2)}\left(4 t^{2}-1\right)^{(n-3) / 2} d t\right] \int_{\mathbf{R}^{n}} f * \breve{f(x)|x|^{2 \beta-n+1} d x}
\end{gathered}
$$

(the first integral converges since we have $\beta<(n-2) / 2$ ).

Now let us estimate

$$
\int_{\mathrm{R}^{n}}\left[\int_{0}^{+\infty} h^{2(k-x)-1}\left(\int_{\mathrm{R}}\left|u_{k}(., h) * F_{\beta, x}(t)\right|^{2} d t\right) d h\right] d x,
$$

where $k$ is the first integer greater than $\alpha$. We have

so

$$
F_{\beta, x} * u_{k}(., h)(\tau)=\iint_{\mathbf{R} \times S^{n-1}}|t|^{\beta} f(x-t y) u_{k}(\tau-t, h) d t d \sigma(y)
$$

$$
\begin{gathered}
\left.\left|F_{\beta, x} * u_{k}(., h)(\tau)\right|^{2}=\iiint \int_{\mathbf{R}^{2} \times S^{n-1} \times S^{n-1}}\left|t_{1} t_{2}\right|^{\beta} f\left(x-t_{1} y_{1}\right) \overline{f\left(x-t_{2} y_{2}\right.}\right) \\
\times u_{k}\left(\tau-t_{1}, h\right) u_{k}\left(\tau-t_{2}, h\right) d t_{1} d t_{2} d \sigma\left(y_{1}\right) d \sigma\left(y_{2}\right) .
\end{gathered}
$$

Using Lemma 1 we get

$$
\begin{gathered}
\left.\int_{\mathbf{R}}\left|F_{\beta, x} * u_{k}(., h)(\tau)\right|^{2} d \tau=\iiint \int_{\mathbf{R}^{2} \times S^{n-1} \times S^{n-1}}\left|t_{1} t_{2}\right|^{\beta} f\left(x-t_{1} y_{1}\right) \overline{f\left(x-t_{2} y_{2}\right.}\right) \\
\times u_{2 k}\left(t_{1}-t_{2}, 2 h\right) d t_{1} d t_{2} d \sigma\left(y_{1}\right) d \sigma\left(y_{2}\right) .
\end{gathered}
$$

If we set $A(\beta, h)=\iint_{\mathbf{R}^{n} \times \mathbf{R}}\left|F_{\beta, x} * u_{k}(., h)(\tau)\right|^{2} d x d \tau$ and $\varphi(x)=f * \check{f(x)}$ we get $A(\beta, h)=\iiint \int_{\mathbf{R}^{2} \times S^{n-1} \times S^{n-1}}\left|t_{1} t_{2}\right|^{\beta} \varphi\left(t_{2} y_{2}-t_{1} y_{2}\right) u_{2 k}\left(t_{1}-t_{2}, 2 h\right) d t_{1} d t_{2} d \sigma\left(y_{1}\right) d \sigma\left(y_{2}\right)$.

By Lemma 2 we obtain

$$
A(\beta, h)
$$

$$
=C \iint_{\mathrm{R}^{2}}\left|t_{1} t_{2}\right|^{\beta} u_{2 k}\left(t_{1}-t_{2}, 2 h\right)\left[\int_{\mathbf{R}^{n}} \varphi(x) \frac{\left[\Delta\left(|x|, t_{1}, t_{2}\right)\right]^{n-3}}{\left(\left|t_{1} t_{2}\right| \cdot|x|\right)^{n-2}} \chi\left(|x|, t_{1}, t_{2}\right) d x\right] d t_{1} d t_{2} .
$$


Using homogeneity properties we get

where

$$
A(\beta, h)=C \int_{\mathbf{R}^{n}} \varphi(x)|x|^{2(\beta-k)+1-n} K(2 h /|x|) d x,
$$

$$
K(\tau)=\iint_{\mathbf{R}^{2}}\left|t_{1} t_{2}\right|^{\beta-n+2} u_{2 k}\left(t_{1}-t_{2}, \tau\right)\left(\Delta\left(1, t_{1}, t_{2}\right)\right)^{n-3} \chi\left(1, t_{1}, t_{2}\right) d t_{1} d t_{2}
$$

We shall show later that we have $|K(\tau)| \leqq C /\left(1+\tau^{2 k+1}\right)$. This being granted we have

$$
\begin{gathered}
\int_{0}^{+\infty} h^{2(k-\alpha)-1} A(\beta, h) d h \leqq C \int_{\mathbf{R}^{n}}|\varphi(x)||x|^{2(\beta-k)-n+1}\left[\int_{0}^{+\infty} \frac{h^{2(k-\alpha)-1}}{1+\left((2 h /|x|)^{2 k+1}\right)} d h\right] d x \\
\leqq C\left[\int_{0}^{\infty} \frac{\tau^{2(k-\alpha)-1}}{1+\tau^{2 k+1}} d \tau\right]\left[\int_{\mathbf{R}^{n}}|\varphi(x)||x|^{2(\beta-\alpha)-n+1} d x\right]
\end{gathered}
$$

The first integral converges and collecting both estimates we get

$$
\int_{\mathbf{R}^{n}}\left\|F_{\beta, x}\right\|_{B_{2}^{\alpha}, 2}^{2}(\mathbf{R}) d x \leqq C \int_{\mathbf{R}^{n}}|f * \check{f}(x)||x|^{2(\beta-\alpha)-n+1}\left(1+|x|^{2 \alpha}\right) d x .
$$

We now have to prove the estimate of $K(\tau)$. By change of variables we get

$$
K(\tau)=C \iint_{\left\{(s, t) \in \mathbf{R}^{2} ;\left(t^{2}-1\right)\left(1-s^{2}\right)>0\right\}}\left|t^{2}-s^{2}\right| \beta-n+2 u_{2 k}(s, \tau)\left|\left(t^{2}-1\right)\left(1-s^{2}\right)\right|^{(n-3) / 2} d s d t
$$

Let us set

$$
L(s)= \begin{cases}\left(1-s^{2}\right)^{(n-3) / 2} \int_{1}^{+\infty}\left(t^{2}-s^{2}\right)^{\beta-n+2}\left(t^{2}-1\right)^{(n-3) / 2} d t \quad \text { if } \quad|s|<1 \\ \left(s^{2}-1\right)^{(n-3) / 2} \int_{0}^{1}\left(s^{2}-t^{2}\right)^{\beta-n+2}\left(1-t^{2}\right)^{(n-3) / 2} d t \quad \text { if } \quad|s|>1\end{cases}
$$

Both integrals converge because $\beta<(n-2) / 2$ and $n \geqq 2$.

$L$ is a $C^{\infty}$ function on $]-1,1$ [ and by Lemma 3 it is integrable in a neighbourhood of -1 and of 1 (because $\beta>-1$ ). In addition, when $|s|$ tends to infinity, we have $L(s)=O\left(s^{2 \beta-n+1}\right)$ so $L$ is integrable.

We have $K(\tau)=C \int_{-\infty}^{+\infty} u_{2 k}(s, \tau) L(s) d s$, so when $s$ tends to zero, $K(\tau)$ tends, save for a multiplicative factor, to the $2 k^{\text {th }}$ deriyative of $L$ at the origin.

In addition $|K(\tau)| \leqq C \int_{-\infty}^{+\infty} \tau\left(\tau^{2}+s^{2}\right)^{-k-1}|L(s)| d s$, thus $K(\tau)=O\left(|\tau|^{-2 k-1}\right)$, when $|\tau|$ tends to infinity. And the proof is complete.

\section{Proof of the Corollaries}

Proof of the first Corollary.

The hypothesis $f \in L^{p}\left(\mathbf{R}^{n}\right) \cap L^{q}\left(\mathbf{R}^{n}\right)$ implies $f * \tilde{f} \in L^{r}\left(\mathbf{R}^{n}\right) \cap L^{s}\left(\mathbf{R}^{n}\right)$ where $r$ and $s$ are defined by $1 / r=2 / p-1,1 / s=2 / q-1$.

More precisely $\|f * \widetilde{f}\|_{L^{r}\left(\mathbf{R}^{n}\right)} \leqq\|f\|_{L^{p}\left(\mathbf{R}^{n}\right)}^{2}$ and $\|f * f\|_{L^{s}\left(\mathbf{R}^{n}\right)} \leqq\|f\|_{L^{a}\left(\mathbf{R}^{n}\right)}^{2}$. 
Let us denote by $r^{\prime}$ and $s^{\prime}$ the conjugate exponents. We have

$$
\begin{gathered}
\int_{\mathbf{R}^{n}}\left\|F_{\beta, x}\right\|_{B_{2}^{\alpha}, 2}^{2}(\mathbf{R}) d x \leqq C\|f\|_{p}^{2}\left(\int_{|x|>1}|x|^{(2 \beta-n+1) r^{\prime}} d x\right)^{1 / r^{\prime}} \\
+C\|f\|_{q}^{2}\left(\int_{|x|<1}|x|^{(2 \beta-2 \alpha-n+1) s^{\prime}} d x\right)^{1 / s^{\prime}} .
\end{gathered}
$$

The first integral converges if $\beta<n(1 / p-1 / 2)-1 / 2$, the second if $\alpha<\beta+1 / 2-$ $n(1 / q-1 / 2)$. So we get a result if

and

$$
-1<\beta<\inf ((n-2) / 2, n(1 / p-1 / 2)-1 / 2)
$$

$$
0<\alpha<\beta+1 / 2-n(1 / q-1 / 2) \text {. }
$$

Proof of Corollary 2.

Let $f$ be a function in $L^{q}\left(\mathbf{R}^{n}\right)$, let us multiply $f$ by the characteristic function of a ball and use Corollary 1 with $p=1$ : if $\alpha$ lies in $] 1 / 2,(n-1) / 2-n(1 / q-1 / 2)[$ one can choose a suitable $\beta$. We conclude using the inclusion $B_{2}^{\alpha, 2}(\mathbf{R}) \subset \Lambda_{z-1 / 2}(\mathbf{R})$.

\section{Proof of Theorem 2}

We shall first prove the following inequality.

Lemma 4. Let $Q$ denote a cube in $\mathbf{R}^{n}$ with diameter equal to 1. Then

$$
\int_{Q}\left\|F_{\mathbf{x}}\right\|_{B_{p}^{\alpha}, 1}^{p} d x \leqq C_{p, \alpha} \int_{\mathbf{R}^{n}}|f(x)|^{p} d x, \quad f \in \mathscr{S}\left(\mathbf{R}^{n}\right),
$$

if $n \geqq 3, n /(n-1)<p<\infty$ and $\alpha<1 / p$.

We need the following notation. Choose $\psi \in C_{0}^{\infty}\left(\mathbf{R}^{n}\right)$ such that supp $(\psi) \subset$ $\{\xi: 1 / 2<|\xi|<2\}$ and

$$
\sum_{v=-\infty}^{\infty} \psi\left(2^{-v} \xi\right)=1, \quad \xi \neq 0 .
$$

Set $\psi_{v}(\xi)=\psi\left(2^{-v} \xi\right), v \in \mathbf{Z}$, and let $\varphi$ and $\varphi_{v}, v=1,2, \ldots$, be defined by $\hat{\varphi}=\psi$ and $\hat{\varphi}_{v}=\psi_{v}$. Here the Fourier transform $\hat{\varphi}$ is defined by

$$
\hat{\varphi}(\xi)=\int_{\mathbf{R}} e^{-i \xi t} \varphi(t) d t
$$

It follows that $\varphi_{v}(t)=2^{v} \varphi\left(2^{v} t\right), v=1,2, \ldots$ We also define $\varphi_{0}$ by setting $\hat{\varphi}_{0}=1-\sum_{1}^{\infty} \psi_{v}$. Then the norm in the Besov space $B_{p}^{\alpha, q}$ is given by

$$
\|f\|_{B_{p}^{\alpha, q}}=\left(\sum_{v=0}^{\infty} 2^{v \alpha q}\left\|\varphi_{v} * f\right\|_{p}^{q}\right)^{1 / q}, \quad 1 \leqq p, q \leqq \infty, \quad \alpha>0 .
$$

Here \|\|$_{p}$ denotes the norm in $L^{p}(\mathbf{R})$ and we make the usual modification for $q=\infty$. 
We observe that it suffices to prove the lemma and the theorem with $B_{p}^{\alpha, 1}$ replaced by $B_{p}^{\alpha, p}$. This follows from a well-known application of Hölder's inequality:

$$
\begin{aligned}
\left\|F_{x}\right\|_{B_{p}^{\alpha, 1}} & =\sum_{0}^{\infty} 2^{v(\alpha-r)} 2^{v r}\left\|\varphi_{y} * F_{x}\right\|_{p} \leqq\left(\sum_{0}^{\infty} 2^{v(\alpha-r) p^{\prime}}\right)^{1 / p^{\prime}}\left(\sum_{0}^{\infty} 2^{v r p}\left\|\varphi_{v} * F_{x}\right\|_{p}^{p}\right)^{1 / p} \\
& \leqq C_{p, \alpha}\left\|F_{x}\right\|_{B_{p}^{r, p}}, \text { where } \alpha<r<1 / p \text { and } 1 / p+1 / p^{\prime}=1 .
\end{aligned}
$$

Proof of Lemma 4. Let $p$ and $\alpha$ satisfy the conditions in the lemma. We shall prove the inequalities

and

$$
\int_{Q}\left\|\varphi_{0} * F_{x}\right\|_{p}^{p} d x \leqq C_{p} \int_{\mathbf{R}^{n}}|f(x)|^{p} d x
$$

$$
\int_{\mathbf{R}^{n}}\left(\sum_{v=1}^{\infty} 2^{v \alpha p}\left\|\varphi_{v} * F_{x}\right\|_{p}^{p}\right) d x \leqq C_{p, \alpha} \int_{\mathbf{R}^{n}}|f(x)|^{p} d x
$$

for $f \in \mathscr{S}\left(\mathbf{R}^{n}\right)$. It is clear that the lemma is a consequence of (3) and (4). We have

$$
\begin{aligned}
& \qquad \varphi_{v} * F_{x}(u)=\int_{\mathbf{R}} \varphi_{v}(u-t) F_{x}(t) d t=\int_{S^{n-1}}\left(\int_{0}^{\infty} \varphi_{v}(u-t) f\left(x-t y^{\prime}\right) d t\right) d \sigma\left(y^{\prime}\right) \\
& =\int_{\mathbf{R}^{n}} \varphi_{v}(u-|y|) f(x-y)|y|^{-n+1} d y=\varphi_{v, u} * f(x), \quad x \in \mathbf{R}^{n}, \quad u \in \mathbf{R}, \quad v=0,1,2, \ldots, \\
& \text { where } \varphi_{v, u}(y)=\varphi_{v}(u-|y|)|y|^{-n+1} .
\end{aligned}
$$

We first prove (3). Since

$$
\begin{gathered}
\left\|\varphi_{0} * F_{x}\right\|_{p} \leqq\left\|\varphi_{0}\right\|_{1}\left\|F_{x}\right\|_{p} \text { it is sufficient to prove that } \\
\int_{Q}\left\|F_{x}\right\|_{p}^{p} d x \leqq C_{p} \int_{\mathbf{R}^{n}}|f(x)|^{p} d x .
\end{gathered}
$$

Using the Minkowski inequality we obtain

$$
\begin{gathered}
\left(\int_{Q}\left\|F_{x}\right\|_{p}^{p} d x\right)^{1 / p}=\left(\iint_{Q \times \mathbf{R}^{+}}\left|\int_{S^{n-1}} f\left(x-t y^{\prime}\right) d \sigma\left(y^{\prime}\right)\right|^{p} d x d t\right)^{1 / p} \\
\leqq \int_{S^{n-1}}\left(\iint_{Q \times \mathbf{R}^{+}}\left|f\left(x-t y^{\prime}\right)\right|^{p} d x d t\right)^{1 / p} d \sigma\left(y^{\prime}\right) .
\end{gathered}
$$

Let $\delta$ denote the diameter of $Q$. We then have

$$
\begin{aligned}
& \iint_{Q \times \mathbf{R}^{+}}\left|f\left(x-t y^{\prime}\right)\right|^{p} d x d t=\sum_{k=0}^{\infty} \int_{3 k \delta}^{3(k+1) \delta}\left(\int_{Q}\left|f\left(x-t y^{\prime}\right)\right|^{p} d x\right) d t \\
& \quad=\int_{0}^{3 \delta}\left(\sum_{k=0}^{\infty} \int_{Q}\left|f\left(x-(t+3 k \delta) y^{\prime}\right)\right|^{p} d x\right) d t \leqq 3 \delta \int_{\mathbf{R}^{n}}|f(x)|^{p} d x
\end{aligned}
$$

for every $y^{\prime} \in S^{n-1}$. (6) follows from this estimate and hence (3) is proved.

We now prove (4) and first observe that

$$
\left\|\varphi_{v, u}\right\|_{L^{1}\left(\mathbf{R}^{n}\right)}=\int_{\mathbf{R}^{n}}\left|\varphi_{v}(u-|y|)\right||y|^{-n+1} d y=C \int_{\mathbf{R}}\left|\varphi_{v}(t)\right| d t=C, \quad v=1,2, \ldots
$$

For the Fourier transform $\hat{\varphi}_{v, u}$ of $\varphi_{v, u}$ we then obtain the estimate

$$
\left\|\hat{\varphi}_{v, u}\right\|_{L^{\infty}\left(\mathbf{R}^{n}\right)} \leqq C, \quad v=1,2, \ldots ; \quad u \in \mathbf{R} .
$$


We shall also prove that

$$
\left\|\hat{\varphi}_{v, u}\right\|_{L^{\infty}\left(R^{n}\right)} \leqq C\left(2^{v}|u|\right)^{-(n-1) / 2}, \quad 2^{v}|u| \geqq 1 .
$$

For $u<0$ this follows from the inequality

$$
\begin{aligned}
& \left\|\hat{\varphi}_{v, u}\right\|_{L^{\infty}\left(\mathbf{R}^{n}\right)} \leqq\left\|\varphi_{v, u}\right\|_{L^{1}\left(\mathbf{R}^{n}\right)}=C \int_{0}^{\infty} 2^{v}\left|\varphi\left(2^{v}(u-t)\right)\right| d t \\
= & C \int_{-\infty}^{2^{v_{u}}}|\varphi(t)| d t \leqq C \int_{2^{v}|u|}^{\infty}(1+t)^{-N} d t \leqq C\left(2^{v}|u|\right)^{-N^{\prime}},
\end{aligned}
$$

where $N$ and $N^{\prime}$ are large positive numbers. For $u>0$ we set $a=2^{v} u$ and assume $a \geqq 1$.

Performing a change of variable $x=u y$ we have

$$
\hat{\varphi}_{v, u}(\xi)=\int_{\mathbf{R}^{n}} e^{-i \xi \cdot x} \varphi_{v}(u-|x|)|x|^{-n+1} d x=\int_{\mathbf{R}^{n}} e^{-i u \xi \cdot y} a \varphi(a(1-|y|))|y|^{-n+1} d y .
$$

Hence

$$
\left\|\hat{\varphi}_{v, u}\right\|_{L^{\infty}\left(\mathbb{R}^{n}\right)}=\|J\|_{L^{\infty}\left(\mathbf{R}^{n}\right)}, \quad \text { where } \quad J(\xi)=\int_{\mathbf{R}^{n}} e^{-i \xi \cdot y} a \varphi(a(1-|y|))|y|^{-n+1} d y .
$$

Assuming $|\xi| \geqq a / 2$ and invoking the well-known estimate

we obtain

$$
\hat{\sigma}(\xi)=\int_{S^{n-1}} e^{-i \xi \cdot y} d \sigma(y)=O\left(|\xi|^{-(n-1) / 2}\right), \quad|\xi| \rightarrow \infty,
$$

$$
\begin{gathered}
|J(\xi)|=\left|\int_{0}^{\infty} a \varphi(a(1-t)) \hat{\sigma}(t \xi) d t\right| \\
\leqq \int_{1 / 2}^{3 / 2} a|\varphi(a(1-t))||\hat{\sigma}(t \xi)| d t+C \int_{|v| \geqq 1 / 2} a|\varphi(a v)| d v \\
\leqq C \int_{1 / 2}^{3 / 2} a|\varphi(a(1-t))|(t|\xi|)^{-(n-1) / 2} d t+\int_{|v| \geqq a / 2}|\varphi(v)| d v \leqq C a^{-(n-1) / 2} .
\end{gathered}
$$

For $|\xi|<a / 2$ we use the fact that $\hat{\varphi}(t)$ vanishes for $|t| \leqq 1 / 2$ and get

$$
\begin{gathered}
|J(\xi)| \leqq\left|\int_{S^{n-1}}\left(\int_{-\infty}^{\infty} e^{-i t \xi^{\prime} \cdot y^{\prime}} a \varphi(a(1-t)) d t\right) d \sigma\left(y^{\prime}\right)\right|+C \int_{-\infty}^{0} a|\varphi(a(1-t))| d t \\
\leqq \int_{S^{n-1}}\left|\varphi\left(\xi \cdot y^{\prime} \mid a\right)\right| d \sigma\left(y^{\prime}\right)+C \int_{1}^{\infty} a|\varphi(a v)| d v \leqq C \int_{a}^{\infty}|\varphi(t)| d t \leqq C a^{-N}
\end{gathered}
$$

where $N$ is a large positive number. Thus (9) is proved.

We let \|\|$_{M_{p}}$ denote the norm in the space $M_{p}\left(\mathbf{R}^{n}\right)$ of Fourier multipliers on $L^{p}\left(\mathbf{R}^{n}\right), 1 \leqq p \leqq \infty$. It follows from (7) that

$$
\left\|\hat{\varphi}_{v, u}\right\|_{M_{\infty}} \leqq C,
$$

and from (8) and (9) we conclude that

$$
\left\|\hat{\varphi}_{v, u}\right\|_{M_{2}} \leqq C\left(1+2^{v}|u|\right)^{-(n-1) / 2} .
$$

Interpolation between $p=2$ and $p=\infty$ yields

$$
\left\|\hat{\varphi}_{v, u}\right\|_{M_{p}} \leqq C\left(1+2^{v}|u|\right)^{-(n-1) / p}, \quad 2 \leqq p \leqq \infty .
$$


By duality we also obtain

$$
\left\|\hat{\varphi}_{v, u}\right\|_{M_{p}} \leqq C\left(1+2^{v}|u|\right)^{-(n-1)(p-1) / p}, \quad 1 \leqq p \leqq 2 .
$$

We now use (10) and (11) to prove (4). Denoting the left hand side of (4) by $B$ we have

$$
\begin{gathered}
B=\sum_{v=1}^{\infty} 2^{v \alpha p} \int_{\mathbf{R}}\left(\int_{\mathbf{R}^{n}}\left|\varphi_{v, u} * f(x)\right|^{p} d x\right) d u \\
\leqq \sum_{v=1}^{\infty} 2^{v \alpha p}\left(\int_{\mathbf{R}}\left\|\hat{\varphi}_{v, u}\right\|_{M_{p}}^{p} d u\right)\left(\int_{\mathbf{R}^{n}}|f(x)|^{p} d x\right) .
\end{gathered}
$$

We denote the first integral on the above right hand side by $I_{p, v}$. We shall prove that

$$
I_{p, v} \leqq C_{p} 2^{-v},
$$

from which (4) follows, since $\alpha<1 / p$. For $p \geqq 2$, (10) yields

$$
I_{p, v} \leqq C \int_{0}^{\infty}\left(1+2^{v} u\right)^{-n+1} d u=C 2^{-v} \int_{0}^{\infty}(1+u)^{-n+1} d u=C 2^{-v},
$$

where we used the assumption $n \geqq 3$. For $n /(n-1)<p<2$ we have $(n-1)(p-1)>1$ and from (11) it follows that

$$
I_{p, v} \leqq C \int_{0}^{\infty}\left(1+2^{v} u\right)^{-(n-1)(p-1)} d u=C 2^{-v} \int_{0}^{\infty}(1+u)^{-(n-1)(p-1)} d u=C_{p} 2^{-v} .
$$

We conclude that (4) holds and the proof of the lemma is complete.

Proof of Theorem 2. It is sufficient to prove that if $Q$ is a cube with diameter 1 and $f \in L^{p}\left(\mathbf{R}^{n}\right)$ then

$$
\int_{Q}\left\|F_{x}\right\|_{B_{p}^{\alpha, p}}^{p_{\alpha}} d x \leqq C_{p, \alpha} \int_{\mathrm{R}^{n}}|f(x)|^{p} d x .
$$

This can be proved by use of the fact that (5) holds for almost every $x \in \mathbf{R}^{n}$ if $f \in L^{p}\left(\mathbf{R}^{n}\right)$, but one can also argue as follows. We may assume that $f$ is non-negative and let $\left(f_{k}\right)_{1}^{\infty}$ denote a non-decreasing sequence of step functions tending to $f$ almost everywhere. It follows from the proof of Lemma 4 that (13) holds with $f$ replaced by $f_{k}$ and $F_{x}$ by the corresponding function $F_{x, k}$. Fatou's lemma yields

$$
\begin{gathered}
\int_{\varepsilon}^{N} \lim _{k \rightarrow \infty}\left(F_{x}(t)-F_{x, k}(t)\right) d t \leqq \varliminf_{k \rightarrow \infty} \int_{\varepsilon}^{N}\left(F_{x}(t)-F_{x, k}(t)\right) d t \\
\leqq \varepsilon^{-n+1} \varliminf_{k \rightarrow \infty} \int_{\varepsilon}^{N}\left(F_{x}(t)-F_{x, k}(t)\right) t^{n-1} d t \\
=\varepsilon^{-n+1} \varliminf_{k \rightarrow \infty} \int_{\varepsilon<|y|<N}\left(f(x-y)-f_{k}(x-y)\right) d y=0
\end{gathered}
$$

for $0<\varepsilon<N$. We conclude that for every $x \in \mathbf{R}^{n}, F_{x}(t)=\lim _{k \rightarrow \infty} F_{x, k}(t)$ for almost every $t$. We have

$$
\int_{Q}\left\|F_{x, k}\right\|_{B_{p}^{\alpha, p}}^{p_{\alpha}} d x \leqq C_{p, \alpha} \int_{\mathrm{R}^{n}}\left|f_{k}(x)\right|^{p} d x
$$


and hence also

$$
\int_{Q}\left\|F_{x, k}\right\|_{p}^{p} d x \leqq C_{p} \int_{\mathbf{R}^{n}}\left|f_{k}(x)\right|^{p} d x .
$$

Letting $k$ tend to infinity in (15) we obtain

$$
\int_{Q}\left\|F_{x}\right\|_{p}^{p} d x \leqq C_{p} \int_{\mathbf{R}^{n}}|f(x)|^{p} d x
$$

and it follows that $F_{x} \in L^{p}(\mathbf{R})$ and $\lim _{k \rightarrow \infty}\left\|F_{x}-F_{x, k}\right\|_{p}=0$ for almost every $x$. As a consequence we also have $\lim _{k \rightarrow \infty}\left\|\varphi_{v} * F_{x, k}\right\|_{p}=\left\|\varphi_{v} * F_{x}\right\|_{p}, v=0,1,2, \ldots$, for almost every $x$. An application of Lebesgue's theorem on dominated convergence yields

$$
\lim _{k \rightarrow \infty} \int_{Q}\left\|\varphi_{\nu} * F_{x, k}\right\|_{p}^{p} d x=\int_{Q}\left\|\varphi_{\nu} * F_{x}\right\|_{p}^{p} d x
$$

and letting $k$ tend to infinity in (14) we obtain (13). The proof of the theorem is complete.

\section{References}

1. Brenner, P., Thomée, V., and WAHLBIN, L. B., Besov spaces and applications to difference methods for initial value problems. Lecture Notes in Mathematics 434, Springer-Verlag 1975.

2. Stein, E. M., Maximal functions: Spherical means. Proc. Nat. Acad. Sci. USA, 73 (1976), $2174-5$.

3. Talbleson, M. H., On the theory of Lipschitz spaces of distributions on Euclidean $n$-space $I$. J. Math. Mech., 13 (1964), 407-479.

Received April 26, 1977

J. Peyrière

Université de Paris VII

U. E. R. de Mathématiques

2, Place Jussieu

F-75 221 Paris Cedex 05

France

P. Sjölin

University of Stockholm

Dept. of Mathematics

Box 6701

S-11385 Stockholm

Sweden 